\title{
Author Correction: Giant optical anisotropy in a quasi-one-dimensional crystal
}

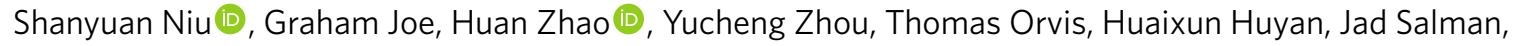
Krishnamurthy Mahalingam, Brittany Urwin, Jiangbin Wu, Yang Liu, Thomas E. Tiwald, Stephen B. Cronin, Brandon M. Howe, Matthew Mecklenburg, Ralf Haiges, David J. Singh, Han Wang, Mikhail A. Kats and Jayakanth Ravichandran

Correction to: Nature Photonics https://doi.org/10.1038/s41566-018-0189-1, published online 18 June 2018.

In the version of this Letter initially published, the identification of light polarization directions (i.e., parallel or perpendicular to the $c$ axis) in optical spectroscopy and ellipsometry results was reversed.

As a result, the directions specified in Fig. 3a,c,d have been corrected. In Fig. 3a,c, from top-down, perpendicular and parallel symbols have now been swapped with each other.

In Fig. 3a, from top-down, perpendicular and parallel symbols on the green and orange color keys, respectively, are now swapped with each other (parallel, top, green; perpendicular, orange, bottom). In Fig. $3 \mathrm{c}$ from top-down, perpendicular and parallel symbols, respectively, on trace labels are similarly now swapped with each other in both instances. In Fig. $3 \mathrm{~d}$, direction of traces are now flipped $180^{\circ}$ on the $y$-axis.

In Fig. 3 caption, in the sentence reading "a,b, Infrared transmission (a) and reflection (b) spectra for incident light polarized perpendicular (dark green) and parallel (orange) to the $c$ axis" the colors "dark green" and "orange" should be swapped to now read: "a,b, Infrared transmission (a) and reflection (b) spectra for incident light polarized perpendicular (orange) and parallel (dark green) to the $c$ axis"

Additionally, discussion of perpendicular and parallel directions in paragraph 6, third and fourth sentences, respectively, were corrected as follows:

"When the polarization was parallel to the $c$ axis, the absorption edge was observed at $4.5 \mu \mathrm{m}(0.27 \mathrm{eV})$. However, when the polarization was perpendicular to the $c$ axis, the absorption edge was blueshifted to $1.6 \mu \mathrm{m}(0.76 \mathrm{eV})$."

The original Letter has been corrected online.

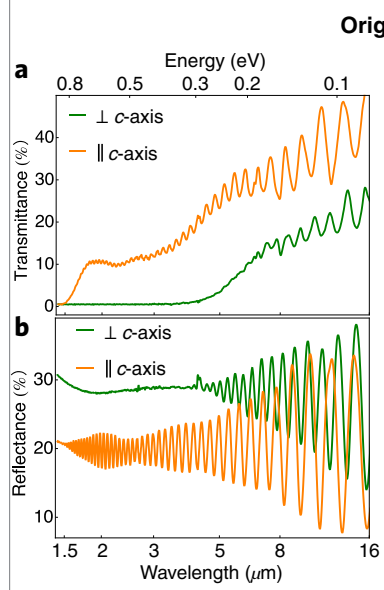

Original
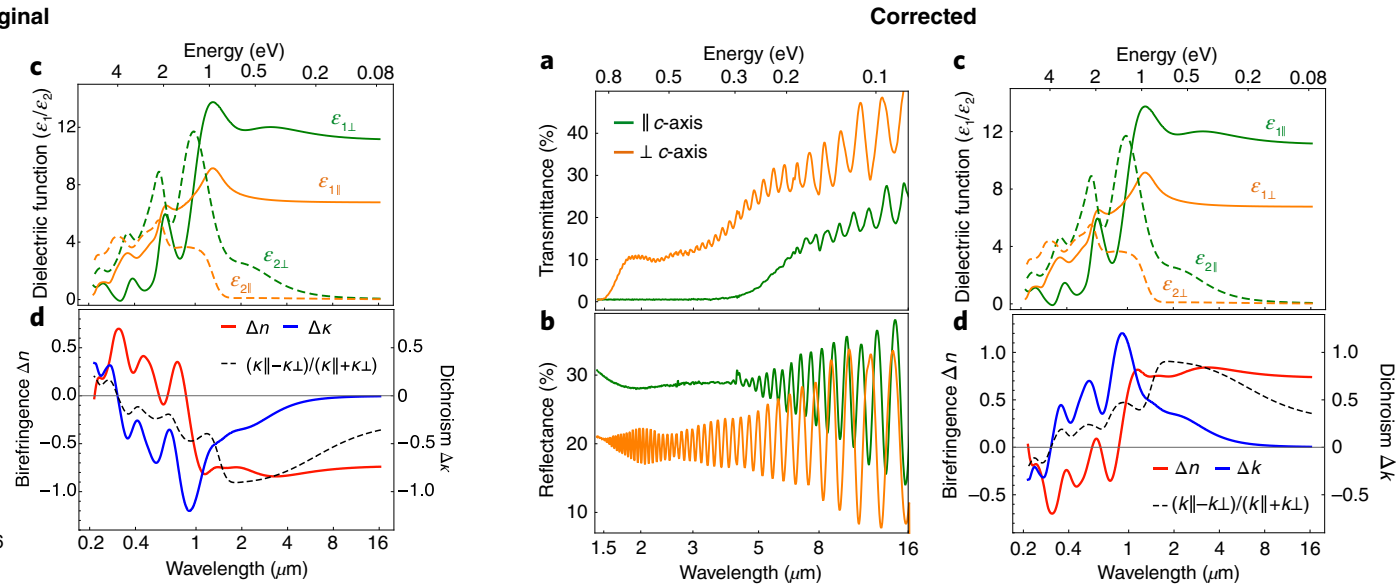

Fig. 3 | Original and Corrected.

Published online: 8 September 2021

https://doi.org/10.1038/s41566-021-00875-y

(C) The Author(s), under exclusive licence to Springer Nature Limited 2021 